\title{
SINGULAR FREE BOUNDARY PROBLEMS AND LOCAL BIFURCATION THEORY*
}

\author{
A. M. STUART $\dagger$
}

\begin{abstract}
A constructive method applicable to the solution of a wide class of free boundary problems is presented. A solution-dependent transformation technique is introduced. By considering a singular limit of the transformation, a related problem, to which local bifurcation theory may be applied, is derived. By inverting the (near singular) mapping between the two problems, an expression for solutions of the original problem is obtained.

The method is illustrated by the study of a singularly perturbed elliptic equation. Approximate solutions are constructed and the validity of the approximations established by means of the Contraction Mapping Theorem.
\end{abstract}

Key words. free boundary problems, bifurcation theory, singular limits

AMS(MOS) subject classifications. 34A08, 34B15, 35B32, 35R35

1. Introduction. In this paper we consider elliptic boundary value problems of the form

$$
\Delta u+\mu H(u-1) f(u)=0 \quad \text { in } \Omega
$$

with

$$
u=0 \quad \text { on } \delta \Omega \text {. }
$$

Here $\Omega$ is the unit ball in $\mathbb{R}^{n}$ centered at the origin. We shall analyze this problem in the singular limit $\mu \rightarrow \infty$. The function $H(\cdot)$ is the Heaviside unit step function satisfying

$$
H(y)=0, \quad y \leqq 0, \quad H(y)=1, \quad y>0 .
$$

The analogue of this problem, when the forcing term is continuous, has been widely studied in the limit $\mu \rightarrow \infty$ (see, for example, [4]).

We consider the case of the bifurcation parameter $\mu>0$. We assume that $f(u)>0$ for $u \geqq 1$, normalize $f(u)$ so that $f(1)=1$ and assume that $f(u) \in C^{3}$ in some neighborhood of $u=1$. The problem is of free boundary type since determination of the sets on which $u(x)=1$ is necessary to solve the problem.

We seek nonnegative solutions possessing the symmetry of the ball and obtain radial solutions $u(x)$ satisfying

$$
\frac{d^{2} u}{d x^{2}}+\frac{(n-1)}{x} \frac{d u}{d x}+\mu H(u-1) f(u)=0
$$

with

$$
\frac{d u}{d x}(0)=u(1)=0
$$

The maximum principle shows that $d u / d x<0$ for $x \in(0,1)$. We note that for semilinear elliptic problems with continuous forcing terms in a ball in $\mathbb{R}^{n}$ all positive solutions are necessarily radial and monotonic decreasing (see [5]).

${ }^{*}$ Received by the editors August 3, 1987; accepted for publication (in revised form) February 26, 1988. This work was supported by the Science and Engineering Research Council of the United Kingdom.

$\dagger$ Oxford University, Mathematical Institute, 24-29 St. Giles, Oxford, OX1 3LB, United Kingdom. Present address, School of Mathematics, University of Bath, Bath BA2 7AY, United Kingdom. 
We present a new, constructive, approach to the solution of (1.1), (1.2) for the cases $n=1$ and $n=2$, which applies to arbitrary nonlinearities $f(u)$. We also deduce rigorous bounds on the errors in the approximate solution. In summary, we show that as $\mu \rightarrow \infty$, solutions of (1.1), (1.2) approach a scaled Green function for the domain (with the scaling ensuring that $\|u\|_{\infty}=1$ ). Furthermore, we characterize the approach to the singular solutions explicitly. The main results are summarized in Corollaries 5.1 and 8.1, concerning the cases $n=1$ and $n=2$, respectively. From an applied mathematical standpoint, such an approach is important since approximate solutions are invaluable as starting points for numerical (continuation) procedures and for local stability calculations.

Although $u \equiv 0$ satisfies (1.1), (1.2), all nontrivial solutions must satisfy $\|u\|_{\infty}>1$. Consequently local bifurcation theory is not directly applicable to the problem. In fact, bifurcation in (1.1), (1.2) occurs from $\mu=\infty$. This phenomenon is discussed in [12] for problems similar to (1.1), (1.2) with a continuous forcing term. In [12], the limiting form of the solution as $\mu \rightarrow \infty$ is zero. However, as $\mu \rightarrow \infty$ in (1.1), (1.2), the solution approaches a scaled Green function and so the problem is highly singular. We describe a transformation technique that captures this singularity and enables us to study a regular problem to which local bifurcation theory is directly applicable.

In $\S 2$ we outline the method presented here in a general context. Sections 3-5 are concerned with the case $n=1$. Section 3 describes the transformations appropriate to (1.1), (1.2). In $\S 4$ we demonstrate that a branch of solutions to (1.1), (1.2) bifurcates from $\mu=\infty,\|u\|_{\infty}=1$ and construct formal series approximations in the neighborhood of the bifurcation point. The free boundary problem is singular in this limit in the sense that the set on which $u>1$ shrinks to zero as $\mu \rightarrow \infty$. We prove convergence of the series approximation and bound the errors by means of the Contraction Mapping Theorem in $\S 5$. Sections $6-8$ concern the case $n=2$. The analysis is similar to that presented for $n=1$ and the details are omitted.

The problem defined by (1.1), (1.2) has been studied previously for $n=1$ by Nistri [8] and Douchet [3] in the case $f(u)>0$ for $u \geqq 1$. Their work employs an analytic shooting technique and, while global, is nonconstructive and, in addition, relies heavily on the fact that solutions of (1.1), (1.2) are monotonic decreasing so that $\|u\|_{\infty}=u(0)$. Our approach is local and constructive and is motivated by work of Berger and Fraenkel [1] described in the following section. The method has been applied in a formal sense to two-point boundary value problems that are not symmetric about the origin (see [9a], [9b] for an example of an application to traveling combustion waves).

Partial differential equations with discontinuous forcing terms have applications in the study of a number of biological and chemical processes which, when viewed on an appropriate timescale, exhibit switchlike behavior. In particular, we are motivated by the study of porous medium combustion where, for small driving gas velocities, the time-dependent analogue of (1.1), (1.2) defines the initial evolution of temperature $u$ in the combustible solid medium before the consumption of reactant becomes appreciable [10], [13]. The parameter $\mu$ represents a scaled heat of reaction. The discontinuity represents the sharp division between regions of chemical reaction and regions of frozen chemistry, which occurs at large activation energies. Experiments indicate that $f(u)$ is proportional to $u^{2}-$ see [9a], [9b] and the references therein.

In addition to this specific application, nonlinear partial differential equations are frequently approximated by simpler problems in which the nonlinearity is replaced by a piecewise continuous profile that is simpler to analyze. Such an approach is employed by Rinzel and Keller in the analysis of the propagation of nerve impulses [11]; (see also Terman [14], [15]). Thus problem (1.1), (1.2) is important not only as a model 
for porous medium combustion, but in its own right since it is a prototype for the study of a wider class of free boundary problems described by problem (P) below.

2. Background and outline of the method. The method described in this paper is applicable to a wide class of nonlinear eigenvalue problems of free boundary type. Specifically, we consider the problem of finding pairs $(\underline{u}, \mu) \in B \times \mathbb{R}$, where $B$ is some Banach space appropriate to the problem, satisfying

$$
\begin{aligned}
& L \underline{u}+\mu H(\underline{a} \cdot \underline{u}+1) \underline{f}(\underline{u})=\underline{0} \quad \text { in } \Omega \subseteq \mathbb{R}^{n}, \\
& D \underline{u}=\underline{0} \quad \text { on } \delta \Omega .
\end{aligned}
$$

Here $L$ is a differential operator and $D$ defines the boundary conditions, which may involve derivatives of $\underline{u}, \underline{f}(\underline{u})$ is some (smooth) vector-valued function and $\underline{a}$ is a constant vector.

We assume that the reduced problem

$$
\begin{array}{ll}
L \underline{u}=\underline{0} & \text { in } \Omega \subseteq \mathbb{R}^{n}, \\
D \underline{u}=\underline{0} & \text { on } \delta \Omega
\end{array}
$$

has the unique solution $\underline{u} \equiv 0$.

Clearly there is no bifurcation of nontrivial solution branches of $(\mathrm{P})$ from the trivial solution since solutions $\underline{u}$ of arbitrarily small supremum norm must have $\underline{a} \cdot \underline{u}<1$ and consequently satisfy $(R)$, which has only the unique trivial solution $\underline{u} \equiv \underline{0}$. Thus we may ask the question whether it is possible to develop a local constructive approach to the solution of problem $(P)$. Since local bifurcation theory is not directly applicable to this problem, the answer to the question is not straightforward.

We introduce a transformation technique, applicable to many problems of the form (P), which enables the techniques of local bifurcation theory to be used. For simplicity we will consider the case $n=1$ or problems for $n>1$, which may be reduced to problems of the form (P) with $n=1$ by symmetry considerations.

The basic idea of the method is that if $L \underline{u}=\underline{0}$ can be integrated explicitly, then (P) may be converted to a problem of the form

( $\left.\mathrm{P}^{*}\right) \quad L \underline{u}+\underline{f}(\underline{u})=\underline{0}$ in $\Omega^{*} \subset \Omega \subseteq \mathbb{R}$ and continuity conditions $D^{*} \underline{u}=\underline{0}$ on $\delta \Omega^{*}$.

Here $\Omega^{*}$ defines the set on which $\underline{a} \cdot \underline{u}>1$, and $D^{*}$ is determined by imposing the required degree of continuity on $\underline{u}$ and its derivatives along $\delta \Omega^{*}$, where $\underline{a} \cdot \underline{u}=1$. Note that $\delta \Omega^{*}$ is unknown and determined by the continuity conditions.

By mapping $\Omega^{*}$ onto a unit interval, a transformed problem $\left(\mathrm{P}^{* *}\right)$ is obtained, which, in the singular limit as $\Omega^{*} \rightarrow \varnothing$ (the empty set) possesses a trivial solution (or family of trivial solutions) with $\underline{a} \cdot \underline{u}=1$. The mapping between $\left(\mathrm{P}^{*}\right)$ and $\left(\mathrm{P}^{* *}\right)$ is, of course, singular and noninvertible when $\Omega^{*}=\varnothing$, so that the trivial solutions of $\left(\mathrm{P}^{* *}\right)$ satisfying $\underline{a} \cdot \underline{u}=1$ do not correspond to solutions of $\left(\mathrm{P}^{*}\right)$. However, by applying local bifurcation theory, constructing nontrivial solutions of $\left(\mathrm{P}^{* *}\right)$ and mapping back to $\left(\mathrm{P}^{*}\right)$ series solutions of $\left(\mathrm{P}^{*}\right)$, and hence of $(\mathrm{P})$, may be constructed.

The fundamental idea underlying this work is that problem $(\mathrm{P})$ simplifies considerably in the limit as the set $\Omega^{*}$, on which $\underline{a} \cdot \underline{u}>1$, shrinks to zero. This important concept was first introduced in the paper of Berger and Fraenkel [1] and has been of great value in the study of elliptic free boundary problems (see, for example, Keady and Norbury [7]).

The work of Berger and Fraenkel, however, is concerned solely with the case $n=2$ and $L$ a second-order elliptic operator, and employs a variational approach. Problems of the form (P), which may be reduced to the study of ordinary differential equations 
and which do not necessarily possess a variational structure, can be tackled by working directly with the governing differential equations. This approach is related to, but somewhat different from, that of Berger and Fraenkel. It has been employed in [9] to establish the existence of traveling wave solutions to the fourth-order partial differential equations governing porous medium combustion. It is the purpose of this paper to illustrate the method by recourse to the slightly simpler, but fundamental, problem defined by (1.1), (1.2).

3. The transformation; the case $n=1$. In this section we transform (1.1), (1.2) for $n=1$ into a form to which local bifurcation theory is directly applicable. We define the unique point $s \in[0,1)$ by

$$
u(s)=1 .
$$

We seek solutions $u(x) \in C^{2}[0,1]$ except at $x=s$ where $d^{2} u / d x^{2}$ is discontinuous. Integrating (1.1) subject to (1.2) and (3.1), we obtain

$$
u=\frac{1-x}{1-s} \text { for } s<x<1
$$

Thus, imposing continuity of $u$ and $d u / d x$ at $x=s$, and symmetry of $u(x)$ about $x=0$, we obtain the following nonlinear eigenvalue problem.

Find $(u(x), s, \mu) \in C^{2}[0, s] \times \mathbb{R}^{2}$ satisfying

$$
\begin{aligned}
& \frac{d^{2} u}{d x^{2}}+\mu f(u)=0, \\
& \frac{d u}{d x}(0)=0, \quad u(s)=1, \\
& \frac{d u}{d x}(s)=\frac{-1}{(1-s)} .
\end{aligned}
$$

This is a specific case of problem $\left(\mathrm{P}^{*}\right)$ described in $\S 2$. Provided that solutions of (P1*) satisfy $u(x)>1$ for $x \in[0, s)$ and $s \in[0,1)$ they correspond to genuine solutions of (1.1), (1.2).

As described in $\S 2$, we map the interval $0<x<s$ onto a unit interval and consider the singular limit $s \rightarrow 0$. In addition we rescale the bifurcation parameter by defining $\lambda=\mu s$. We set $z=x / s$, and, for convenience, define $w(z)=u(s z)-1$. We obtain, for $' \equiv d / d z$, the following nonlinear eigenvalue problem.

Find $(w(z), s, \lambda) \in C^{2}[0,1] \times \mathbb{R}^{2}$ satisfying

$$
\left(\mathrm{P} 1^{* *}\right) \quad w^{\prime \prime}+\lambda s f(1+w)=0, \quad w^{\prime}(0)=w(1)=0, \quad w^{\prime}(1)=-s /(1-s) .
$$

This is a specific case of Problem $\left(\mathrm{P}^{* *}\right)$ described in $\S 2$. Provided $s$ is nonzero the mapping between $\left(\mathrm{P} 1^{*}\right)$ and $\left(\mathrm{P} 1^{* *}\right)$ is a bijection so that solutions of $\left(\mathrm{P} 1^{* *}\right)$ satisfying $w(z)>0$ for $z \in[0,1)$ and $s \in(0,1)$ correspond to genuine solutions of (1.1), (1.2).

Notice that $\left(\mathrm{P} 1^{* *}\right)$ possesses the trivial solution $w(z)=s=0$ for all values of the (scaled) bifurcation parameter $\lambda$. Thus, by considering the singular limit of a solution dependent transformation of independent variable, we have created an artificial trivial solution. Since $s=0$ this does not correspond to a genuine solution of (1.1), (1.2); however, by seeking solutions of $\left(\mathrm{P} 1^{* *}\right)$, which bifurcate from the trivial solution $w(z)=s=0$ and mapping back to (1.1), (1.2), we may obtain genuine solutions. 
The transformation $z=x / s$, which maps $0<x<s$ onto a unit interval, is fundamental to the technique described here for all problems of the form $(P)$. The transformation $\lambda=\mu s$ of the bifurcation parameter is, however, specific to problem (1.1), (1.2); the problem tackled in [9a], [9b], for example, does not require a transformation of bifurcation parameter.

4. Formal bifurcation analysis for $\left(\mathbf{P} 1^{* *}\right)$. Let $(\varphi(z), \zeta)$ denote the linearizations of $(w(z), s)$ about $(0,0)$. From $\left(\mathrm{P} 1^{* *}\right)$ we deduce that $(\varphi(z), \zeta)$ satisfies

$$
\varphi^{\prime \prime}+\lambda \zeta=0, \quad \varphi^{\prime}(0)=\varphi(1)=0, \quad \varphi^{\prime}(1)=-\zeta .
$$

This eigenvalue problem (which, on appropriate definition of function spaces, defines the Fréchet derivative of $\left(\mathrm{P}^{* *}\right)$ with respect to the trivial solution) has a nontrivial solution if and only if $\lambda=1$. Thus we expect bifurcation of a nontrivial solution to $\left(\mathrm{P} 1^{* *}\right)$ at $\lambda=1$.

Since bifurcating solutions are of small amplitude, we seek formal series solutions of $\left(\mathrm{P} 1^{* *}\right)$ with $s \ll 1$. We expand $w(z)$ and the bifurcation parameter $\lambda$ in powers of $s$ in the form

$$
w(z)=\sum_{i=0}^{\infty} s^{i+1} w_{i}(z) \text { and } \quad \lambda=\sum_{i=0}^{\infty} s^{i} \lambda_{i}
$$

Substituting into $\left(\mathrm{P} 1^{* *}\right)$, we obtain

$$
\sum_{i=0}^{\infty} s^{i+1} w_{i}^{\prime \prime}(z)+\sum_{i=0}^{\infty} s^{i+1} \lambda_{i} f\left(1+\sum_{i=0}^{\infty} s^{i+1} w_{i}(z)\right)=0
$$

together with boundary conditions

$$
w_{i}^{\prime}(0)=w_{i}(1)=0 \quad \text { and } \quad w_{i}^{\prime}(1)=-1 .
$$

Equating powers of $s$ in (4.1) we obtain the problems

$$
\begin{aligned}
& w_{0}^{\prime \prime}+\lambda_{0}=0, \\
& w_{1}^{\prime \prime}+\lambda_{1}+\lambda_{0} f_{u}(1) w_{0}=0, \\
& w_{2}^{\prime \prime}+\lambda_{2}+\lambda_{1} f_{u}(1) w_{0}+\lambda_{0} f_{u}(1) w_{1}+\lambda_{0} f_{u u}(1) w_{0}^{2} / 2=0 .
\end{aligned}
$$

Note that each second-order differential equation has three boundary conditions, given by (4.2), the extra one determining the $\lambda_{i}$. Solving these successive problems yields $\lambda_{0}=1$ (as expected from the analysis above) and

$$
\begin{aligned}
& w_{0}(z)=\frac{\left(1-z^{2}\right)}{2}, \\
& w_{1}(z)=\frac{\left(1-z^{2}\right)}{2}+\frac{f_{u}(1)}{24}\left(1-z^{2}\right)^{2}, \\
& \lambda_{1}=1-\frac{f_{u}(1)}{3} \\
& \lambda_{2}=1-\frac{2 f_{u}(1)}{3}+\frac{4\left[f_{u}(1)\right]^{2}}{45}-\frac{f_{u u}(1)}{15}
\end{aligned}
$$

In the next section we prove rigorously that $\left(\mathrm{P} 1^{* *}\right)$ possesses a solution $(w(z), s, \lambda)$ satisfying

$$
\left\|w(z)-w_{0}(z) s-w_{1}(z) s^{2}\right\|_{\infty} \leqq O\left(s^{3}\right) \quad \text { and } \quad\left|\lambda-1-\lambda_{1} s\right| \leqq O\left(s^{2}\right),
$$


for $s \ll 1$, with error constants independent of $z \in[0,1]$. Note that, since $\lambda=\mu s$ and $u(x)=1+w(z)$, this demonstrates that $\mu \rightarrow \infty$ and $\|u\|_{\infty} \rightarrow 1$ as $s \rightarrow 0$.

5. Rigorous bifurcation analysis for $\left(\mathbf{P} 1^{* *}\right)$. We rigorously establish the existence of small amplitude nontrivial solutions of $\left(\mathrm{P}^{* *}\right)$ bifurcating from $w(z)=s=0$ at $\lambda=1$. Furthermore we find explicit approximations to $w(z)$ and for $s \ll 1$ and prove that they are accurate up to terms of $O\left(s^{3}\right)$ and $O\left(s^{2}\right)$, respectively. To achieve these results we employ the Contraction Mapping Theorem. It is possible to establish bifurcation from $\lambda=1$ in the transformed problem $\left(P 1^{* *}\right)$ by application of the known results of bifurcation theory [2] and this is done in [13]. However, the results in [13] rely purely on an analysis of the eigenvalue problem arising from the linearization of (P1**) about the trivial solution. The nonlinear effects, which determine whether the bifurcation in $\left(P 1^{* *}\right)$ is of transcritical or pitchfork type, are not analyzed. The estimates of the solution obtained in this paper are an order of magnitude more accurate than those obtained in [13] and account for the effect of the nonlinearities. The degree of accuracy obtained here is often required for initiating numerical continuation procedures near the bifurcation point or for time-dependent stability calculations [10]. We demonstrate that bifurcation in $\left(\mathrm{P} 1^{* *}\right)$ is transcritical provided that $f_{u}(1)$ is not equal to three. Only the branches of solutions in $\left(\mathrm{P} 1^{* *}\right)$ with $s \in(0,1)$ are relevant to problem (P1).

In order to employ the Contraction Mapping Theorem we formulate $\left(\mathrm{P}^{* *}\right)$ as an integral equation. We denote by $g(z ; y)$ the Green function satisfying the problem

$$
g^{\prime \prime}(z ; y)=\delta(z-y), \quad g^{\prime}(0 ; y)=g(1 ; y)=0,
$$

with solution

$$
\begin{array}{ll}
g(z ; y)=(y-1), & 0<z<y<1, \\
g(z ; y)=(z-1), & 0<y<z<1 .
\end{array}
$$

Thus $\left(\mathrm{P} 1^{* *}\right)$ is equivalent to finding $(w(z), s, \lambda) \in C[0,1] \times \mathbb{R}^{2}$ satisfying

$$
w(z)=-\lambda s \int_{0}^{1} g(z ; y) f(1+w(y)) d y
$$

with the continuity condition on $w^{\prime}(1)$ :

$$
\lambda \int_{0}^{1} f(1+w(y)) d y=(1-s)^{-1} .
$$

Here we have used the fact that $g_{z}(1 ; y)=1$, by $(5.1)$.

By employing the Green function (5.1), (4.3)-(4.5) subject to (4.2) yield the following expressions for $w_{0}, w_{1}, w_{2}, \lambda_{0}, \lambda_{1}$, and $\lambda_{2}$ :

$$
\left\{\begin{array}{l}
w_{0}(z)=-\int_{0}^{1} \lambda_{0} g(z ; y) d y \\
w_{1}(z)=-\int_{0}^{1} g(z ; y)\left[\lambda_{1}+\lambda_{0} f_{u}(1) w_{0}(y)\right] d y \\
w_{2}(z)=-\int_{0}^{1} g(z ; y)\left[\lambda_{2}+\lambda_{1} f_{u}(1) w_{0}(y)+\lambda_{0} f_{u}(1) w_{1}(y)+\lambda_{0} f_{u u}(1) w_{0}^{2}(y) / 2\right] d y
\end{array}\right.
$$




$$
\left\{\begin{array}{l}
\int_{0}^{1} \lambda_{0} d y=1 \\
\int_{0}^{1}\left[\lambda_{1}+\lambda_{0} f_{u}(1) w_{0}(y)\right] d y=1 \\
\int_{0}^{1}\left[\lambda_{2}+\lambda_{1} f_{u}(1) w_{0}(y)+\lambda_{0} f_{u}(1) w_{1}(y)+\lambda_{0} f_{u u}(1) w_{0}^{2}(y) / 2\right] d y=1
\end{array}\right.
$$

Here we have again used the fact that $g_{z}(1 ; y)=1$. We are now in a position to prove the following theorem.

THEOREM 5.1. For each $s>0$, sufficiently small, problem $\left(\mathrm{P} 1^{* *}\right)$ possesses a unique nontrivial solution satisfying

$$
\begin{aligned}
& \left\|w(z)-w_{a}(z)\right\|_{\infty} \leqq \bar{w} s^{3}, \\
& \left|\lambda-\lambda_{a}\right| \leqq \bar{\lambda} s^{2}
\end{aligned}
$$

where $w_{a}(z)=w_{0}(z) s+w_{1}(z) s^{2}, \lambda_{a}=1+\lambda_{1} s+\lambda_{2} s^{2}, \bar{w} / 2=C_{1}=\sup _{z \in[0,1]}\left|w_{2}(z)\right|$ and $\bar{\lambda}=$ $2 C_{1} / 3$. Here $w_{0}(z), w_{1}(z), w_{2}(z), \lambda_{1}$, and $\lambda_{2}$ are defined by (4.6)-(4.9), respectively, and also implicitly by $(5.4 \mathrm{a}),(5.4 \mathrm{~b})$.

Proof. We define the fixeci point mapping:

$$
\begin{aligned}
& v_{n+1}(z)=-\eta_{n} s \int_{0}^{1} g(z, y) f\left(1+v_{n}(y)\right) d y, \\
& \eta_{n+1}=\left[(1-s) \int_{0}^{1} f\left(1+v_{n+1}(y)\right) d y\right]^{-1} .
\end{aligned}
$$

We prove that, for $s$ sufficiently small, this mapping has a unique fixed point $(w(z), \lambda) \in$ $C[0,1] \times \mathbb{R}$ satisfying $(5.5),(5.6)$. Such a fixed point is clearly a solution of $(5.2),(5.3)$, and hence $\left(\mathrm{P} 1^{* *}\right)$. We define $X$ to be the closed subset of $C[0,1] \times \mathbb{R}$ satisfying (5.5), (5.6) and prove that the iteration (5.7), (5.8) maps $X$ into itself and is contractive in the norm of the product Banach space $C[0,1] \times \mathbb{R}$ for elements of $X$. By the Contraction Mapping Theorem [6] this establishes the required result.

First we establish that (5.7), (5.8) maps $X$ into itself. From (5.7) we obtain, by Taylor series expansion,

$$
\begin{aligned}
\left|v_{n+1}(z)-w_{a}(z)\right|= & \mid \eta_{n} s \int_{0}^{1}-g(z ; y)\left[1+f_{u}(1) v_{n}(y)+f_{u u}(1) v_{n}^{2}(y) / 2\right. \\
& \left.+f_{\text {uиu }}(\xi) v_{n}^{3}(y)\right] d y-w_{a}(z) \mid
\end{aligned}
$$

Assuming that $\left(v_{n}(z), \eta_{n}\right) \in X$, we have

$$
\begin{aligned}
&\left|v_{n+1}(z)-w_{a}(z)\right| \leqq\left(s+\lambda_{1} s^{2}+\bar{\lambda} s^{3}+\lambda_{2} s^{3}\right) \int_{0}^{1}-g(z ; y) \\
& \cdot\left[1+s f_{u}(1) w_{0}(y)+s^{2}\left\{f_{u}(1) w_{1}(y)+f_{u u}(1) w_{0}^{2}(y) / 2\right\}+O\left(s^{3}\right)\right] d y-w_{a}(z) \mid
\end{aligned}
$$

where the $O\left(s^{3}\right)$ term is bounded uniformly with respect to $y$. Note that $\xi$ lies between one and $1+v_{n}(y)$; since $f(u) \in C^{3}$ in a neighborhood of $u=1$ we deduce that $\left|f_{\text {uиu }}(\xi)\right|$ is bounded for $s$ sufficiently small, since $\left(v_{n}(z), \eta_{n}\right) \in X$. 
Collecting terms of equal powers of $s$ and employing (5.4) to simplify the expressions, we obtain

$$
\left|v_{n+1}(z)-w_{a}(z)\right| \leqq\left\{\bar{\lambda}\left|\int_{0}^{1} g(z ; y) d y\right|+\left|w_{2}(z)\right|\right\} s^{3}+O\left(s^{4}\right) .
$$

Maximizing over $z \in[0,1]$, using definition (5.1) of $g(z ; y)$, we obtain

$$
\left\|v_{n+1}(z)-w_{a}(z)\right\|_{\infty} \leqq\left(\bar{\lambda} / 2+C_{1}\right) s^{3}+O\left(s^{4}\right),
$$

where $C_{1}$ is defined in the statement of the theorem. For $s$ sufficiently small we eliminate the $O\left(s^{4}\right)$ term and write

$$
\left\|v_{n+1}(z)-w_{a}(z)\right\|_{\infty} \leqq\left(3 \bar{\lambda} / 4+3 C_{1} / 2\right) s^{3} .
$$

Thus, by the definitions of $\bar{\lambda}$ and $\bar{w}$ in the theorem, $v_{n+1}(z)$ satisfies the bound (5.5) whenever $\left(v_{n}(z), \eta_{n}\right) \in X$.

From (5.8) we obtain

$$
\begin{aligned}
\left|\eta_{n+1}-\lambda_{a}\right|= & \left|\left[(1-s) \int_{0}^{1} f\left(1+v_{n+1}(y)\right) d y\right]^{-1}-\lambda_{a}\right| \\
= & \mid\left[1+s+s^{2}+\frac{s^{3}}{(1-s)}\right] \\
& \cdot\left[1+\int_{0}^{1}\left\{v_{n+1}(y) f_{u}(1)+v_{n+1}^{2}(y) f_{u u}(1) / 2\right.\right. \\
+ & \left.\left.v_{n+1}^{3}(y) f_{u u u}(\bar{\xi}) / 6\right\} d y\right]^{-1}-\lambda_{a} \mid .
\end{aligned}
$$

Here $\left|f_{\text {uuu }}(\bar{\xi})\right|$ is bounded for $s$ sufficiently small because $v_{n+1}(z)$ satisfies (5.5).

Since $\left|v_{n+1}(z)-w_{a}(z)\right| \leqq \bar{w} s^{3}$ we have

$$
\begin{aligned}
\left|\eta_{n+1}-\lambda_{a}\right|= & \mid\left[1+s+s^{2}+O\left(s^{3}\right)\right]\left[1+\int_{0}^{1}\left\{w_{0}(y) f_{u}(1) s\right\} d y\right. \\
& \left.+\int_{0}^{1}\left\{w_{1}(y) f_{u}(1)+w_{0}^{2}(y) f_{u u}(1) / 2\right\} s^{2} d y+O\left(s^{3}\right)\right]^{-1}-\lambda_{a} \mid \\
\leqq & \mid\left[1+s+s^{2}+O\left(s^{3}\right)\left[1-\int_{0}^{1}\left\{w_{0}(y) f_{u}(1) s\right\} d y\right.\right. \\
& -\int_{0}^{1}\left\{w_{1}(y) f_{u}(1)+w_{0}^{2}(y) f_{u u}(1) / 2\right\} s^{2} d y \\
& \left.+\left\{\int_{0}^{1} w_{0}(y) f_{u}(1) d y\right\}^{2} s^{2}+O\left(s^{3}\right)\right]-\lambda_{a} \mid .
\end{aligned}
$$

Now,

$$
\lambda_{a}=1+\lambda_{1} s+\lambda_{2} s^{2} .
$$

Using (5.4b) to eliminate $\lambda_{1}$ and $\lambda_{2}$, noting that $\lambda_{0}=1$, we obtain

$$
\begin{aligned}
\lambda_{a}= & +\left[1-\int_{0}^{1} f_{u}(1) w_{0}(y) d y\right] s \\
& +\left[1-\int_{0}^{1} f_{u}(1) w_{0}(y) d y+f_{u}(1) w_{1}(y)+f_{u u}(1) w_{0}^{2}(y) / 2 d y\right. \\
& \left.+\left(\int_{0}^{1} f_{u}(1) w_{0}(y) d y\right)^{2}\right] s^{2} .
\end{aligned}
$$


Substituting this into the upper bound for $\left|\eta_{n+1}-\lambda_{a}\right|$, we obtain

$$
\left|\eta_{n+1}-\lambda_{a}\right| \leqq O\left(s^{3}\right) \text {. }
$$

Thus $\eta_{n+1}$ satisfies (5.6) automatically for $s$ sufficiently small. This completes the proof that (5.7), (5.8) maps $X$ into itself. We now proceed to demonstrate that (5.7), (5.8) defines a contraction.

Consider two sets of iterates $\left(\theta_{n}(z), \alpha_{n}\right)$ and $\left(\phi_{n}(z), \beta_{n}\right)$ satisfying (5.7), (5.8) and contained in $X$. Then

$$
\begin{aligned}
\left|\theta_{n+1}(z)-\phi_{n+1}(z)\right|= & \left|\beta_{n} s \int_{0}^{1} g(z ; y) f\left(1+\phi_{n}(y)\right) d y-\alpha_{n} s \int_{0}^{1} g(z ; y) f\left(1+\theta_{n}(y)\right) d y\right| \\
= & \mid \alpha_{n} s \int_{0}^{1} g(z ; y)\left[f\left(1+\phi_{n}(y)\right)-f\left(1+\theta_{n}(y)\right)\right] d y \\
& +\left(\beta_{n}-\alpha_{n}\right) s \int_{0}^{1} g(z, y) f\left(1+\phi_{n}(y)\right) d y \mid \\
\leqq & \left|\int_{0}^{1} g(z ; y) d y\right|\left\{\alpha_{n} C_{2} s\left\|\theta_{n}(z)-\phi_{n}(z)\right\|_{\infty}+C_{3} s\left|\beta_{n}-\alpha_{n}\right|\right\},
\end{aligned}
$$

where $C_{2}=\max _{z \in[0,1]}\left|f_{u}(1+w(z))\right|$ and $C_{3}=\max _{z \in[0,1]}|f(1+w(z))|$, for $w(z)$ satisfying (5.5).

Maximizing over $z$ and noting that, since $\left(u_{n}(z), \alpha_{n}\right) \in X$, we have $\alpha_{n} \leqq \lambda_{a}+\bar{\lambda} s^{2}$, yielding

$$
\left\|\theta_{n+1}(z)-\phi_{n+1}(z)\right\|_{\infty} \leqq \frac{1}{2}\left\{\left(\lambda_{a}+\bar{\lambda} s^{2}\right) C_{2} s\left\|\theta_{n}(z)-\phi_{n}(z)\right\|_{\infty}+C_{3} s\left|\beta_{n}-\alpha_{n}\right|\right\} .
$$

Also, by (5.8)

$$
\begin{aligned}
\left|\alpha_{n+1}-\beta_{n+1}\right| & =\frac{\left|(1-s)^{-1} \int_{0}^{1}\left\{f\left(1+\phi_{n+1}(y)\right)-f\left(1+\theta_{n+1}(y)\right)\right\} d y\right|}{\left[\int_{0}^{1} f\left(1+\phi_{n+1}(y)\right) d y\right]\left[\int_{0}^{1} f\left(1+\theta_{n+1}(y)\right) d y\right]} \\
& \leqq(1-s)^{-1} C_{2}\left\|\phi_{n+1}-\theta_{n+1}\right\|_{\infty} / C_{4}^{2} .
\end{aligned}
$$

Here $C_{4}=\min _{z \in[0,1]} \mid f(1+w(z)) \|$, for $w(z)$ satisfying (5.5). Since $f(1)=1$ we deduce that $C_{4}$ may be bounded away from zero independently of $s$, sufficiently small.

Combining (5.9) and (5.10) we show that

$$
\left\|\theta_{n+1}(z) \phi_{n+1}(z)\right\|_{\infty}+\left|\alpha_{n+1}-\beta_{n+1}\right| \leq C_{5} s\left\{\left\|\theta_{n}(z)-\phi_{n}(z)\right\|_{\infty}+\left|\alpha_{n}-\beta_{n}\right|\right\},
$$

where

$$
C_{5}=\left\{\frac{1}{2}+\frac{(1-s)^{-1} C_{2}}{2 C_{4}^{2}}\right\} \max \left\{\left(\lambda_{a}+\bar{\lambda} s^{2}\right) C_{2}, C_{3}\right\} .
$$

Since $\|w(z)\|_{\infty}+|\lambda|$ is the appropriate norm for the product Banach space $C[0,1] \times \mathbb{R}$ we deduce that, for $s$ sufficiently small, (5.7), (5.8) define a contraction on $X$. This completes the proof.

COROLlaRY 5.1. For each $s>0$ sufficiently small (1.1), (1.2) with $n=1$ possesses a unique nontrivial solution satisfying

$$
\begin{aligned}
& \sup _{x \in[0,1]}\left|u(x)-u_{a}(x)\right|=\left\|u(x)-u_{a}(x)\right\|_{\infty} \leqq \bar{w} s^{3}, \\
& \left|\mu-\mu_{a}\right| \leqq \bar{\lambda} s,
\end{aligned}
$$


where

$$
\begin{array}{ll}
u_{a}(x)=1+w_{0}(x / s) s+w_{1}(x / s) s^{2} & \text { for } 0<x<s, \\
u_{a}(x)=(1-x) /(1-s) & \text { for } s<x<1, \\
\mu_{a}=s^{-1}+\lambda_{1}+\lambda_{2} s, &
\end{array}
$$

and $w_{0}(z), w_{1}(z), \bar{w}, \lambda_{1}, \lambda_{2}$, and $\bar{\lambda}$ are defined in Theorem 5.1.

Proof. Solutions of $\left(\mathrm{P}^{* *}\right)$ are in a one-to-one correspondence with solutions of (1.1), (1.2) provided that $s \in(0,1)$ and $w(z)>0$ for $z \in[0,1)$. These conditions are satisfied by the solution constructed in Theorem 5.1; mapping back from ( $\left.\mathrm{P} 1^{* *}\right)$ to (1.1), (1.2), we obtain the required result.

Examination of this corollary shows that for $n=1(1.1),(1.2)$ possesses a branch of solutions bifurcating from $\mu=\infty$ and $\|u(x)\|_{\infty}=1$. Furthermore we have characterized the form of the solution accurately in the neighborhood of this bifurcation point. If we extend the solution constructed, by symmetry, to $x \in[-1,1]$ then we find that, as $\mu \rightarrow \infty(s \rightarrow 0), \quad u(x) \rightarrow 2 G(x ; 0)$ where the Green function $G(x, y)$ satisfies $d^{2} G / d x^{2}(x ; y)=\delta(x-y)$ for $x \in[-1]$ and $G( \pm 1 ; y)=0$. Here the scale factor two in front of the Green function ensures that $\|u(x)\|_{\infty} \rightarrow 1$ as $\mu \rightarrow \infty$.

6. The transformation; the case $n=2$. In this section we transform (1.1), (1.2) for $n=2$ into a form to which local bifurcation theory is directly applicable. As in $\S 3$ we define the unique point $s \in[0,1)$ by $u(s)=1$. We seek solutions $u(x) \in C^{2}[0,1]$ except at $x=s$ where $d^{2} u / d x^{2}$ is discontinuous. Integrating (1.1) with $n=2$ subject to (1.2) and (3.1), we obtain

$$
u=\frac{\operatorname{Lnx}}{\operatorname{Ln} s} \text { for } s<x<1 .
$$

Thus, imposing continuity of $u$ and $d u / d x$ at $x=s$, and symmetry of $u(x)$ about $x=0$, we obtain the following nonlinear eigenvalue problem.

Find $(u(x), s, \mu) \in C^{2}[0, s] \times \mathbb{R}^{2}$ satisfying

$$
\begin{aligned}
& \frac{1}{x} \frac{d}{d x}\left(x \frac{d u}{d x}\right)+\mu f(u)=0 \\
& \frac{d u}{d x}(0)=0, \quad u(s)=1, \quad \frac{d u}{d x}(s)=1 / s(\text { Lns }) .
\end{aligned}
$$

This is a specific case of problem $\left(\mathrm{P}^{*}\right)$ described in $\S 2$. Provided that solutions of (P2*) satisfy $u(x)>1$ for $x \in[0, s)$ and $s \in[0,1)$ they correspond to genuine solutions of (1.1), (1.2) with $n=2$.

Again, as described in $\S 2$, we map the interval $0<x<s$ onto a unit interval and consider the singular limit $s \rightarrow 0$. As in $\S 3$ it is necessary to make an additional solution dependent rescaling of the bifurcation parameter by defining $\lambda=-(L n s) s^{2} \mu$. We set $z=x / s$, and, for convenience, define $w(z)=u(s z)-1$. We obtain, for ' $\equiv d / d z$, the following nonlinear eigenvalue problem.

Find $(w(z), \gamma, \lambda) \in C^{2}[0,1] \times \mathbb{R}^{2}$ satisfying

$\left(\mathrm{P} 2^{* *}\right) \quad \frac{1}{z}\left(z w^{\prime}\right)^{\prime}+\gamma \lambda f(1+w)=0, \quad w^{\prime}(0)=w(1)=0, \quad w^{\prime}(1)=-\gamma$,

where $\gamma=-1 /($ Lns $)$.

As in $\S 3(\mathrm{P} 2 * *)$ defines a genuine solution of $(1.1),(1.2)$ with $n=2$ whenever $w(z)>0$ for $z \in[0,1)$ and $s \in(0,1)$. We note that $\left(\mathrm{P} 2^{* *}\right)$ has the trivial solution 
$w(z)=\gamma=0$ for all values of $\lambda$. Again, since $\gamma \rightarrow 0_{+}$corresponds to $s \rightarrow 0_{+}$, the solution does not correspond to a true solution of $\left(\mathrm{P}^{*}\right)$, since the mapping from $\left(\mathrm{P}^{*}\right)$ to $\left(\mathrm{P} 2^{* *}\right)$ is noninvertible when $s=0$. However, as in the case $n=1$, we now apply local bifurcation theory and construct solutions of $\left(\mathrm{P} 2^{* *}\right)$ with $0<\gamma \ll 1$, which correspond to true solutions of (P2).

7. Formal bifurcation analysis for $\left(\mathbf{P 2}{ }^{* *}\right)$. A regular bifurcation argument similar to that in $\S 4$ shows that $\lambda=2$ is the only bifurcation point for $\left(\mathrm{P} 2^{* *}\right)$. Thus we seek formal series solutions of $\left(\mathrm{P} 2^{* *}\right)$ with $\gamma \ll 1$ and expand $w(z)$ and $\lambda$ in the form

$$
w(z)=\sum_{i=0}^{\infty} \gamma^{i+1} w_{i}(z) \text { and } \lambda=\sum_{i=0}^{\infty} \gamma^{i} \lambda_{i} .
$$

Expanding $\left(\mathrm{P} 2^{* *}\right)$ in powers of $\gamma$ and equating coefficients, we obtain the equations

$$
\begin{aligned}
& \frac{1}{z}\left(z w_{0}^{\prime}\right)^{\prime}+\lambda_{0}=0 \\
& \frac{1}{z}\left(z w_{1}^{\prime}\right)^{\prime}+\lambda_{1}+\lambda_{0} f_{u}(1) w_{0}=0 \\
& \frac{1}{z}\left(z w_{2}^{\prime}\right)^{\prime}+\lambda_{2}+\lambda_{1} f_{u}(1) w_{0}+\lambda_{0} f_{u}(1) w_{1}+\lambda_{0} f_{u u}(1) w_{0}^{2} / 2=0,
\end{aligned}
$$

subject to

$$
\begin{array}{ll}
w_{i}^{\prime}(0)=w_{i}(1)=0 \quad \text { for } i \geqq 0, \\
w_{0}^{\prime}(1)=-1 \quad \text { and } \quad w_{i}^{\prime}(1)=0, \quad i \geqq 1 .
\end{array}
$$

Solving these successive problems, with the $\lambda_{i}$ determined by (7.5), we obtain

$$
\begin{aligned}
& w_{0}(z)=\frac{\left(1-z^{2}\right)}{2}, \\
& w_{1}(z)=\frac{f_{u}(1)\left(1-z^{2}\right)^{2}}{16}, \\
& \lambda_{0}=2 \\
& \lambda_{1}=-\frac{f_{u}(1)}{2}, \\
& \lambda_{2}=\frac{11 f_{u}^{2}(1)}{24}-\frac{f_{u u}(1)}{3} .
\end{aligned}
$$

In the next section we prove rigorously that $(\mathrm{P} 2 * *)$ possesses a solution $(w(z), \gamma, \lambda)$ satisfying

$$
\begin{aligned}
& \left\|w(z)-w_{0}(z) \gamma-w_{1}(z) \gamma^{2}\right\|_{\infty} \leqq O\left(\gamma^{3}\right), \\
& \left|\lambda-2-\lambda_{1} \gamma\right| \leqq O\left(\gamma^{2}\right),
\end{aligned}
$$

for $\gamma=-1 /(L n s) \ll 1$, with error constants independent of $z \in[0,1]$.

8. Rigorous bifurcation analysis for $\left(\mathbf{P 2}^{* *}\right)$. We establish rigorously the existence of small amplitude nontrivial solutions of $\left(\mathrm{P} 2^{* *}\right)$ bifurcating from $w(z)=\gamma=0$ at $\lambda=2$. The analysis and conclusions of this section are very similar to those in $\S 5$ for $\left(\mathrm{P} 1^{* *}\right)$ and the details are omitted. 
We denote by $g(z ; y)$ the Green function satisfying the problem

$$
\frac{1}{z}\left(z g^{\prime}(z ; y)\right)^{\prime}=\delta(z-y), \quad g^{\prime}(0 ; y)=g(1 ; y)=0,
$$

with solution

$$
\begin{array}{ll}
g(z ; y)=y(\text { Lny }), & 0<z<y<1, \\
g(z ; y)=y(\operatorname{Ln} z), & 0<y<z<1 .
\end{array}
$$

Thus ( $\left.\mathrm{P} 2^{* *}\right)$ is equivalent to finding $(w(z), \gamma, \lambda) \in C[0,1] \times \mathbb{R}^{2}$ satisfying

$$
w(z)=-\lambda \gamma \int_{0}^{1} g(z ; y) f(1+w(y)) d y
$$

with the continuity condition on $w^{\prime}(1)$ :

$$
\lambda \int_{0}^{1} g_{z}(1 ; y) f(1+w(y)) d y=1 .
$$

By employing the definition of $g(z ; y)$ in (8.1) we may write (7.1)-(7.4) for $w_{0}(z)$, $w_{1}(z)$, and $w_{2}(z)$ as in (5.4a). The matching conditions (7.5) yield

$$
\begin{aligned}
& \int_{0}^{1} g_{z}(1 ; y) \lambda_{0} d y=1, \\
& \int_{0}^{1} g_{z}(1 ; y)\left[\lambda_{1}+\lambda_{0} f_{u}(1) w_{0}(y)\right] d y=0, \\
& \int_{0}^{1} g_{z}(1 ; y)\left[\lambda_{2}+\lambda_{1} f_{u}(1) w_{0}(y)+\lambda_{0} f_{u}(1) w_{1}(y)+\lambda_{0} f_{u u}(1) w_{0}^{2}(y) / 2 f d y=0 .\right.
\end{aligned}
$$

We may now prove the following result.

THEOREM 8.1. For each $\gamma>0$, sufficiently small, problem $\left(\mathrm{P} 2^{* *}\right)$ possesses a unique nontrivial solution satisfying

$$
\begin{aligned}
& \left\|w(z)-w_{a}(z)\right\|_{\infty} \leqq \bar{w} \gamma^{3}, \\
& \left|\lambda-\lambda_{a}\right| \leqq \bar{\lambda} \gamma^{2},
\end{aligned}
$$

where $w_{a}(z)=w_{0}(z) \gamma+w_{1}(z) \gamma^{2}, \lambda_{a}=\lambda_{0}+\lambda_{1} \gamma+\lambda_{2} \gamma^{2}, \bar{w} / 2=C_{6}=\sup _{z \in[0,1]}\left|w_{2}(z)\right|$ and $\bar{\lambda}=4 C_{6} / 3$. Here $w_{0}(z), w_{1}(z), w_{2}(z), \lambda_{0}, \lambda_{1}$, and $\lambda_{2}$ are defined by (7.6)-(7.10), respectively, and also implicitly by (5.4a) and (8.4).

Proof. The proof is nearly identical to that of Theorem 5.1 differing only in the form of the Green function and in the continuity condition on $w^{\prime}(1)$. Consequently we omit the details. We define the fixed point mapping

$$
\begin{aligned}
& v_{n+1}(z)=-\eta_{n} \int_{0}^{1} g(z ; y) f\left(1+v_{n}(y)\right) d y, \\
& \eta_{n+1}=\left[\int_{0}^{1} g_{z}(1 ; y) f\left(1+v_{n+1}(y)\right) d y\right]^{-1} .
\end{aligned}
$$

We prove that, for $s$ sufficiently small, this mapping has a unique fixed point $(w(z), \lambda) \in$ $C[0,1] \times \mathbb{R}$ satisfying (8.5), (8.6). Such a fixed point clearly solves (8.2), (8.3), and hence $\left(\mathrm{P} 2^{* *}\right)$. We define $X$ to be the closed subset of $C[0,1] \times \mathbb{R}$ satisfying $(8.5),(8.6)$ and prove that the iteration (8.7), (8.8) maps $X$ into itself and is contractive in the 
norm of the product Banach space $C[0,1] \times \mathbb{R}$ for elements of $X$. By the Contraction Mapping Theorem [6] this establishes the required result.

Similar manipulations to those in the proof of Theorem 5.1 yield

$$
\left|v_{n+1}(z)-w_{a}(z)\right| \leqq\left\{\bar{\lambda}\left|\int_{0}^{1} g(z ; y) d y\right|+\left|w_{2}(z)\right|\right\} \gamma^{3}+O\left(\gamma^{4}\right) .
$$

From (8.1) we obtain

$$
\left\|v_{n+1}(z)-w_{a}(z)\right\|_{\infty} \leqq\left(\bar{\lambda} / 4+C_{6}\right) \gamma^{3}+O\left(\gamma^{4}\right),
$$

where $C_{6}$ is defined in the statement of the theorem. For $\gamma$ sufficiently small we deduce that

$$
\left\|v_{n+1}(z)-w_{a}(z)\right\|_{\infty}<\left(3 \bar{\lambda} / 8+3 C_{6} / 2\right) \gamma^{3} .
$$

Also, as in the proof of Theorem 5.1, we find that

$$
\left|v_{n+1}-\lambda_{a}\right| \leqq O\left(\gamma^{3}\right) \text {. }
$$

Using the definitions of $\bar{\lambda}$ and $\bar{w}$ in the theorem, (8.9) and (8.10) imply that (8.7), (8.8) maps $X$ into itself. We now proceed to demonstrate that $(8.7),(8.8)$ defines a contraction.

Consider two sets of iterates $\left(\theta_{n}(z), \alpha_{n}\right)$ and $\left(\phi_{n}(z), \beta_{n}\right)$ satisfying (8.7), (8.8) and contained in $X$. Analysis identical to that in the proof of Theorem 5.1 (except that (8.1) now defines $g(z ; y))$ gives

$$
\left\|\theta_{n+1}(z)-\phi_{n+1}(z)\right\|_{\infty} \leqq \frac{1}{4}\left\{\left(\lambda_{a}+\bar{\lambda} s^{2}\right) C_{7} s\left\|\theta_{n}(z)-\phi_{n}(z)\right\|+C_{8} s\left|\beta_{n}-\alpha_{n}\right|\right\}
$$

where $C_{7}=\max _{z \in[0,1]}\left|f_{u}(1+w(z))\right|$ and $C_{8}=\max _{z \in[0,1]}|f(1+w(z))|$ for $w(z)$ satisfying (8.5).

We may also show that

$$
\left|\alpha_{n+1}-\beta_{n+1}\right| \leqq C_{7}\left\|\theta_{n+1}-\phi_{n+1}\right\|_{\infty} / C_{9}^{2},
$$

where $C_{9}=\min _{z \in[0,1]} \mid f(1+w(z)) \|$, for $w(z)$ satisfying (8.5). Since $f(1)=1$ we deduce that $C_{9}$ may be bounded away from zero independently of $s$, sufficiently small.

Combining (8.11) and (12) we show that

$$
\left\|\left(\theta_{n+1}(z)-\phi_{n+1}(z), \alpha_{n+1}-\beta_{n+1}\right)\right\| \leqq C_{10}\left\{\left\|\left(\theta_{n}(z)-\phi_{n}(z), \beta_{n}-\alpha_{n}\right)\right\|\right\}
$$

where $C_{10}=\left\{\frac{1}{4}+C_{7} / 4 C_{9}^{2}\right\} \max \left\{\left(\lambda_{a}+\bar{\lambda} \gamma^{2}\right) C_{7}, C_{8}\right\}$ and $\|(w(z), \lambda)\| \equiv\|w(z)\|_{\infty}+|\lambda|$, the appropriate norm for the product Banach space $C[0,1] \times \mathbb{R}$. We deduce that, for $\gamma$ sufficiently small, (8.7), (8.8) defines a contraction on $X$. This completes the proof.

COROllary 8.1. For each $s>0$ sufficiently small (1.1), (1.2) with $n=2$ possesses a unique nontrivial solution satisfying

$$
\begin{aligned}
& \sup _{x \in[0,1]}\left|u(x)-u_{a}(x)\right|=\left\|u(x)-u_{a}(x)\right\|_{\infty} \leqq-\bar{w} /(\text { Lns })^{3}, \\
& \left|\mu-\mu_{a}\right| \leqq-\bar{\lambda} /(\text { Lns })^{3} s^{2}
\end{aligned}
$$

where

$$
\begin{aligned}
& u_{a}(x)=1-w_{0}(x / s) /(\text { Lns })+w_{1}(x / s) /(\text { Lns })^{2} \text { for } 0<x<s \\
& u_{a}(x)=\operatorname{Ln} x / \text { Lns for } s<x<1 \\
& \mu_{a}=-\left(2-\lambda_{1} /(\text { Lns })+\lambda_{2}(L n s)^{2}\right) /\left(s^{2} \text { Lns }\right),
\end{aligned}
$$

$w_{0}(z), w_{1}(z), \bar{w}, \lambda_{1}, \bar{\lambda}$, and $\lambda_{2}$ are defined as in Theorem 8.1. 
Proof. Solutions of $(\mathrm{P} 2 * *)$ are in a one-to-one correspondence with solutions of (1.1), (1.2) with $n=2$, provided that $s \in(0,1)$ and $w(z)>0$ for $z \in[0,1)$. These conditions are satisfied by the solution constructed in Theorem 8.1; mapping back from $\left(\mathrm{P} 2^{* *}\right)$ to $(1.1),(1.2)$, noting that $\lambda=-(L n s) s^{2} \mu$ and $\gamma=-1 /(L n s)$, we obtain the required result.

Note that, as $\mu \rightarrow \infty(s \rightarrow 0)$, we find that the solution $u(x)$ of (1.1), (1.2) with $n=2$ satisfies $u(x) \rightarrow \operatorname{Lnx} / \operatorname{Lns}$ for $s<x<1$, which is a scaled version of the Green function for the Laplacian in a ball in $\mathbb{R}^{2}$. As $s \rightarrow 0$ this function ceases to be continuous, since $u(s)=1$ and $u(x) \rightarrow 0$ for $x \in(0,1]$. Thus we have essentially constructed a smoothing perturbation of a singular solution. This is the process described as "nonlinear desingularization" in the paper of Berger and Fraenkel [1].

The results of Corollary 8.1 (the case $n=2$ ) are weaker than those of Corollary 5.1 (the case $n=1$ ), since the bound (8.13) merely yields an asymptotic expression for $\mu$ as $s \rightarrow 0$, whereas the bound (5.11) provides a strict upper bound of $O(s)$ on the error in the expression for $\mu$ as $s \rightarrow 0$. The weaker results in two dimensions are caused by the fact that the Green function for the Laplacian in a ball in $\mathbb{R}^{n}$ is a more singular function in two dimensions than in one. For identical reasons the methods described here break down when applied to (1.1), (1.2) in dimensions $n \geqq 3$. Similar difficulties are encountered for $n \geqq 3$ in the alternative approach of Berger and Fraenkel [1].

\section{REFERENCES}

[1] M. S. Berger AND L. E. Fraenkel, Nonlinear desingularisation in certain free boundary problems, Comm. Math. Phys., 77 (1980), pp. 149-172.

[2] S-N. Chow ANd J. HALE, Methods of Bifurcation Theory, Springer-Verlag, New York, 1982.

[3] J. DOUCHET, The number of positive solutions of a nonlinear problem with discontinuous nonlinearity, Proc. Roy. Soc. Edinburgh Sect. A, 90 (1981), pp. 281-291.

[4] P. C. FIFE, Semilinear elliptic boundary value problems with small parameters, Arch. Rational Mech. Anal., 52 (1973), pp. 205-232.

[5] B. GIDAS, W-M. NI, AND L. NiRENBERG, Symmetry and related properties via the maximum principle, Comm. Math. Phys., 68 (1979), pp. 209-243.

[6] D. H. Griffel, Applied Functional Analysis, Ellis Horwood, Chichester, 1979.

[7] G. KeAdy AND J. NORbury, A semilinear elliptic eigenvalue problem, parts I and II, Proc. Roy. Soc. Edinburgh Sect. A, 87 (1980), pp. 65-109.

[8] P. Nistri, Positive solutions of a nonlinear eigenvalue problem with discontinuous nonlinearity, Proc. Roy. Soc. Edinburgh Sect. A, 83 (1979), pp. 133-145.

[9a] J. NORBURY AND A. M. STUART, Travelling combustion waves in a porous medium, part I, SIAM J. Appl. Math., 48 (1988), pp. 155-169.

[9b] - Travelling combustion waves in a porous medium, part II, SIAM J. Appl. Math., 48 (1988), pp. 374-392.

[10] - Parabolic free boundary problems arising in porous medium combustion, IMA J. Appl. Math., 39, (1987), pp. 241-257.

[11] J. RinzEL AND J. B. Keller, Travelling wave solutions of a nerve conduction equation, Biophysical J., 13 (1973), pp. 1313-1337.

[12] S. Rosenblat AND S. Davis, Bifurcation from infinity, SIAM J. Appl. Math., 37 (1979), pp. 1-19.

[13] A. M. STUART, The mathematics of porous medium combustion, in Nonlinear Diffusion Equations and their Equilibrium States, L. A. Peletier, W.-M. Ni, and J. Serrin, eds., Springer-Verlag, Berlin, New York, 1988.

[14] D. TERMAN, A free boundary problem arising from a bistable reaction-diffusion equation, SIAM J. Math. Anal., 14 (1983), pp. 1107-1129.

[15] —- A free boundary arising from a model for nerve conduction. J. Differential Equations, 58 (1985), pp. 345-363. 